OPEN

SUBJECT AREAS:

NANOPHOTONICS AND

PLASMONICS

MICRO-OPTICS

Received

14 April 2014

Accepted

2 June 2014

Published

25 June 2014

Correspondence and requests for materials should be addressed to

E.D. (emiliano.

descrovi@polito.it)

\section{Focusing and Extraction of Light mediated by Bloch Surface Waves}

\author{
Angelo Angelini ${ }^{1,2}$, Elsie Barakat ${ }^{3}$, Peter Munzert ${ }^{4}$, Luca Boarino ${ }^{2}$, Natascia De Leo ${ }^{2}$, Emanuele Enrico², \\ Fabrizio Giorgis' ${ }^{1}$, Hans Peter Herzig ${ }^{3}$, Candido Fabrizio Pirri' \& Emiliano Descrovi ${ }^{1}$
}

'Department of Applied Science and Technology, Politecnico di Torino, Corso Duca degli Abruzzi 24, Torino IT-10129, Italy, ${ }^{2}$ Nanofacility Piemonte, Istituto Nazionale di Ricerca Metrologica, Strada delle Cacce 91, Torino IT-10135, Italy, ${ }^{3}$ Optics \& Photonics Technology Laboratory, Ecole Polytechnique Fédérale de Lausanne, rue de la Maladière 71b, Neuchâtel CH-2002, Switzerland, ${ }^{4}$ Fraunhofer Institute for Applied Optics and Precision Engineering IOF, Albert-Einstein-Str. 7, Jena DE-07745, Germany.

The control of emission from localized light sources is an objective of outstanding relevance in nanophotonics. In a recent past, a large number of metallic nanostructures has been proposed to this end, wherein plasmonic modes are exploited as energy carriers on a subwavelength scale. As an interesting alternative, we present here the use of surface modes on patterned dielectric multilayers to deliver electromagnetic power from free-space to localized volumes and vice versa. Thanks to this low-loss energy transfer, proper periodic ring structures are shown to provide a subwavelength focusing of an external radiation onto the multilayer surface. By reciprocity, the radiated power from emitters within the ring center is shown to be efficiently beamed in the free-space, with a well-controlled angular divergence. This mechanism overcomes some important limitations involved in the all-plasmonic approach, while opening new opportunities for hybrid devices in photon management applications such as optical sensing and lighting.

$\mathrm{T}$ he transfer of electromagnetic energy between free-space and localized volumes has deserved intense research efforts during the last years. The nano-antenna paradigm well illustrates this general concept at optical frequencies ${ }^{1}$, especially in cases wherein the energy delivery is resonantly assisted by plasmonic modes ${ }^{2}$. Individual antennas constituted by single or few nano-scatterers ${ }^{3-7}$ can convert deeply confined energy into freely propagating radiation (and vice versa) according to some angularly anisotropy, i.e. the antenna directivity. On the other hand, when multiple antennas are spatially arranged in proper arrays, the overall directivity can be greatly improved, at the cost of a general lack of energy localization in the near-field ${ }^{8-11}$. However, periodic structures can also act as mere couplers for propagating surface plasmons ${ }^{12}$ that can be further localized on a subwavelength scale by several additional means ${ }^{13-15}$. Therefore, a system based on periodic structures can still support an energy conversion process between localized volumes and free-space, when mediated by propagating surface plasmons ${ }^{16}$. Previously proposed arrangements, which exploit circular gratings coupled to plasmonic nano-cavities, have been demonstrated to exhibit both a strong energy confinement capability and a good directivity of the radiated electromagnetic field ${ }^{17,18}$. Unfortunately, the use of surface plasmons as energy carriers in these systems suffers from ohmic losses that reduce the efficiency of the overall mechanism.

In order to overcome the limitation above, we propose here an alternative approach wherein the energy transfer between free-space and localized volumes is mediated by surface modes on dielectric multilayers. The low absorption exhibited by the multilayer materials makes the surface modes propagating over long distances, in the order of hundreds of micrometers ${ }^{19}$ in a very wide spectral range. In the specific arrangement presented here, we demonstrate that a structure constituted by a circular grating on a planar multilayer shows optical features similar to the well-known plasmonic ring antennas ${ }^{18}$ with much narrower resonances. In fact, despite the underlying physics is radically different, the multilayered grating structure can out-couple radiation emitted by localized sources with a very high directivity into the free-space and in-couple and focus surface waves on a subwavelength scale, according to the limitations imposed by an all-dielectric structure. In addition to the experimental evidence for these two effects, we also provide a quantitative estimation of the fluorescence collection enhancement obtained by exploiting the antenna effect in an exemplary low-Numerical Aperture optical system. 

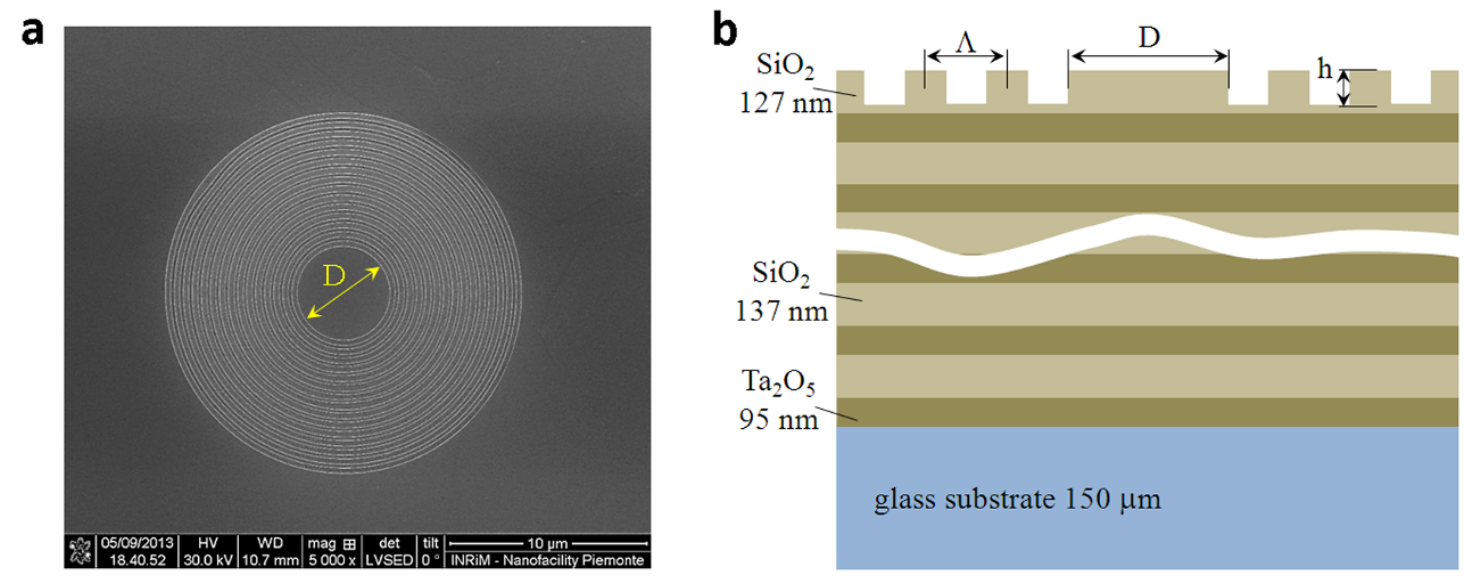

Figure $1 \mid$ Sample structure. (a), Scanning Electron Microscope image of the circular grating fabricated on the multilayer structure. (b), sketch of the cross-sectional view of the patterned multilayer. The stack sequence is glass- $\left[\mathrm{Ta}_{2} \mathrm{O}_{5}-\mathrm{SiO}_{2}\right] \times 6-\mathrm{Ta}_{2} \mathrm{O}_{5}-\mathrm{SiO}_{2}$-air for the "regular" layout, while it is glass$\left[\mathrm{Ta}_{2} \mathrm{O}_{5}-\mathrm{SiO}_{2}\right] \times 10-\mathrm{Ta}_{2} \mathrm{O}_{5}-\mathrm{SiO}_{2}$-aifor the "low leakage" layout. The grating period is $\Lambda=520 \mathrm{~nm}$ and the inner spacer diameter can be either $\mathrm{D}=5 \mu \mathrm{m}$ or $\mathrm{D}=8 \mu \mathrm{m}$. The height of the grating is $\mathrm{h}=100 \mathrm{~nm}$.

\section{Results}

BWS coupling and focusing. The sample structure is sketched in Figure 1 and detailed in the Method section. Very briefly, it consists of a dielectric multilayer constituted by a periodic stack of Silica and Tantalia layers, whereon circular gratings are fabricated. The multilayer is such that TE-polarized surface modes (also called Bloch Surface Waves -BSWs-) can be sustained at wavelengths below $600 \mathrm{~nm}$, as illustrated by the angularly resolved reflectivity map shown in Supplementary Figure S1(a). The BSW field distribution across the multilayer is quite strongly confined on the surface, where an intense near-field enhancement occurs ${ }^{20}$ (Supplementary Figure S1(b)). This effect is due to two reasons: (i) the BSW is a surface-bound state (i.e. the BSW wavevector is larger than $2 \pi / \lambda$ and therefore it does not leak in air), (ii) the BSW dispersion curve lays within a forbidden band of the underlying multilayer. Given the stack sequence described in the Method section, two kinds of multilayers are considered here: a "regular" layout consisting of 16 total layers and a "low leakage" layout, consisting of 22 layers. The main difference between the two layouts is in the total amount of power that can be resonantly transferred from/to the BSW to/from propagating radiation in the glass substrate. Although the band structure of these two kinds of multilayers is substantially similar (and so is the BSW dispersion curve), the "low leakage" layout sustains BSW with lower losses due to leakage into the substrate.

The circular grating is etched on the multilayer top layer in such a way that a ring pattern is arranged with a spatial period $\Lambda=520 \mathrm{~nm}$ around a flat inner spacer. The total thickness of the grating is about $100 \mathrm{~nm}$. The grating vector is radially oriented with respect to the circular grating center, and has a module $\mathrm{K}=2 \pi / \Lambda=12.08 \mu \mathrm{m}^{-1}$ that is very close to the BSW wavevector in the wavelength range between $532 \mathrm{~nm}$ and $590 \mathrm{~nm}$ wavelength.

When an external radiation of a specific wavelength $\lambda$ is impinging on the circular grating from air, it undergoes diffraction according to the Bragg's law. The first-order $(+1)$ diffracted radiation has a wavevector component $\mathrm{k}_{\mathrm{T}}{ }^{+1}$ parallel to the multilayer surface as given by $\mathrm{k}_{\mathrm{T}}^{+1}=\mathrm{k}_{\mathrm{T}}{ }^{0}+\mathrm{K}$, where $\mathrm{k}_{\mathrm{T}}{ }^{0}$ is the wavevector component of the incident radiation parallel to the multilayer surface. When $\mathrm{k}_{\mathrm{T}}^{+1}$ matches the BSW wavevector, energy coupling between the incident radiation and the surface mode can occur. For an incidence radiation with wavelength $\lambda=532 \mathrm{~nm}$ (as used here), the BSW cannot be coupled at a perfectly normal incidence, but an incidence angle of about 2.3 degrees is required.

In a recent paper it has been demonstrated that a linear grating can couple BSW on a planar multilayer ${ }^{21}$. In the case of the ring structures shown here, the grating has a curvature radius and an in-plane focusing effect can be expected for the grating-coupled BSW, in analogy to surface plasmons ${ }^{22,23}$. This effect has been observed by locally illuminating a portion of a circular grating fabricated on a "regular" multilayer and observing the leakage radiation associated to BSW as collected with an interference leakage radiation microscope (detailed in the Method section and illustrated in Supplementary Figure S2). Starting from the illuminated area, a BSW is coupled and then focused in a central region of the inner spacer surrounded by the circular grating. Experimental amplitude and phase maps of the focused BSW are shown in Supplementary Figure S3. The appearance of a pair of phase dislocations in the BSW focal region supports the observation of the focusing effect ${ }^{24}$.

More interestingly, when the circular grating is homogeneously and fully illuminated with the laser beam, the coupling to BSW can occur according to the local spatial orientation of the grating. A circular BSW converging toward the center can be then produced. In order to provide momentum matching between BSW and the incident radiation through the grating, the angular spread of the laser beam can be slightly adjusted (see Methods section). Experimental amplitude and phase maps of focused BSW are presented in Fig. 2(a,b). If the laser is linearly polarized, BSW can be coupled only from locations of the rings wherein a polarization matching condition is satisfied. Specifically, the TE-polarization of BSW allow efficient coupling only from those regions having grating vector perpendicular to the laser polarization. In the present case, for a $y$-polarized incident beam, the leakage radiation associated to the focused BSW has a two-lobe symmetric distribution oriented along the $\mathrm{x}$-axis. When the laser polarization is rotated, the observed pattern is rotated accordingly (see Supplementary Figure S4). The BSW focusing effect produces a subwavelength central lobe in the spacer center, with an estimated Full Width Half Height of about $220 \mathrm{~nm}$ (Fig. 2(c)).

We observe that the amplitude profile shows a fringe pattern with a spatial frequency doubled as compared to the wavefront frequency appearing in Fig. 2(b). This is due to an interference effect by two counter-propagating BSWs. The phase cross-section along the horizontal line sketched in Fig. 2(b) shows a sawtooh profile indicating the two counter-propagating BSWs converging at the grating center (Fig. 2(d)). The existence of these two counter-propagating BSWs can be better appreciated by considering the Fourier spectrum amplitude calculated from the complex field (obtained from measured amplitude and phase) along the horizontal line (Fig. 2(e)). The Fourier spectrum amplitude exhibits two main contributions peaked at spatial frequencies $\mathrm{f}_{\mathrm{x}}= \pm 2.025 \mu \mathrm{m}^{-1}$, corresponding to the cal- 

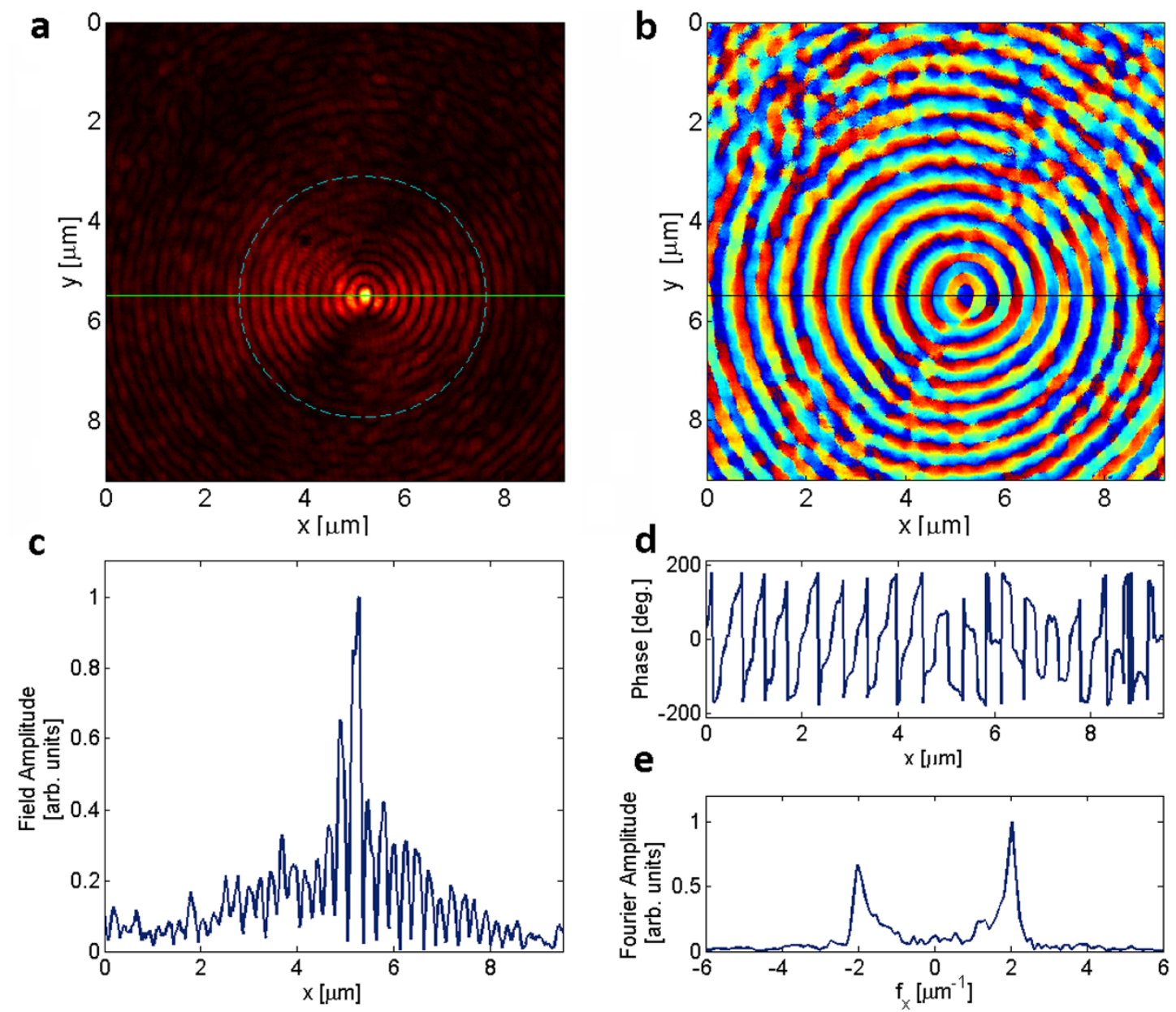

Figure $2 \mid$ Amplitude and Phase of leaking BSWs. (a), amplitude and (b), phase distribution of the leakage radiation associated to a converging laser BSW. Coupling is performed by illuminating a circular grating $(\mathrm{D}=5 \mu \mathrm{m}, \Lambda=520 \mathrm{~nm})$ on a "regular" multilayer with a linearly polarized (y-direction) laser $(\lambda=532 \mathrm{~nm})$. The dashed circle indicates the boundary on the inner spacer. (c), cross-sectional field amplitude profile along the horizontal line shown in (a). (d), cross-sectional field phase profile and (e) its corresponding Fourier spectrum amplitude along the horizontal line shown in (b).

culated BSW spatial frequency obtained from the BSW dispersion shown in Supplementary Figure S1(a).

The benefits from BSW focusing can be appreciated in cases wherein a selective enhanced fluorescence excitation is desired in the spacer center only. Fluorescence measurements are performed after incubation of AlexaFluor 546-labelled protein A on the sample. In this way, a rather photostable fluorescent layer can be homogeneously provided over a large surface area. Figure 3 shows direct plane fluorescence images of a ring structure homogeneously illuminated by a collimated laser. The setup used for these measurements is described in the Method section. The illumination area is extending well beyond the lateral size of the circular grating. Moreover, it is worth to underline that fluorescence collection is performed here by a NA $=0.2$ objective working in air, in such a way that the BSWcoupled fluorescence leaking into the substrate is prevented to contribute to the image formation.

When the incident laser is circularly polarized, a fluorescence image as shown in Fig. 3(a) is collected. We observe some fluorescence emitted from the whole grating surrounding a bright spot located in the spacer center. Although the organic emitter is homogeneously distributed on the whole multilayer surface, at the outside of the ring structure the background fluorescence is rather low. By taking a (normalized) intensity profile along the horizontal line drawn in Fig. 3(a), the intensity contrast across the structure can be better appreciated. The bright central spot shows a fluorescence intensity that is roughly 10 times higher than the fluorescence com- ing from the outside of the ring (Fig. 3(b)). This increase in fluorescence is produced by the BSW coupling and focusing from the laser illumination. Since the spacer region is flat and rather wide, any physical accumulation of the emitting molecules on the surface can be excluded.

In case the incident laser radiation is linearly polarized, the fluorescence pattern shows a two-lobed shape, whose orientation rotates depending on the orientation of the laser polarization (Fig. 3(c-e)). By comparing results with the interferometric analysis of Fig. 2, we can conclude that the fluorescence distribution presented above mainly represents the fluorescence trace of the BSW coupled from the laser. However, it is well known that the fluorescence emission can in turn couple to surface modes ${ }^{25}$. This effect is separately addressed in the next sections.

Fluorescence beaming. In this section, the coupling of localized emitters to BSW on the multilayered ring structure is considered. In order to investigate the coupling of fluorescence into the multilayer modes and the corresponding propagation directions out of the sample, a microscopy technique based on Back Focal Plane (BFP) imaging ${ }^{26,27}$ is employed (details in the Methods section). Illumination is provided as a focused laser beam.

Figure 4(a) shows a typical BFP fluorescence image collected from a planar area with no adjacent structures. The observed bright rings correspond to fluorescence coupled to TE and TM modes of the multilayer and leaking into the glass substrate ${ }^{20}$. In particular, fluor- 

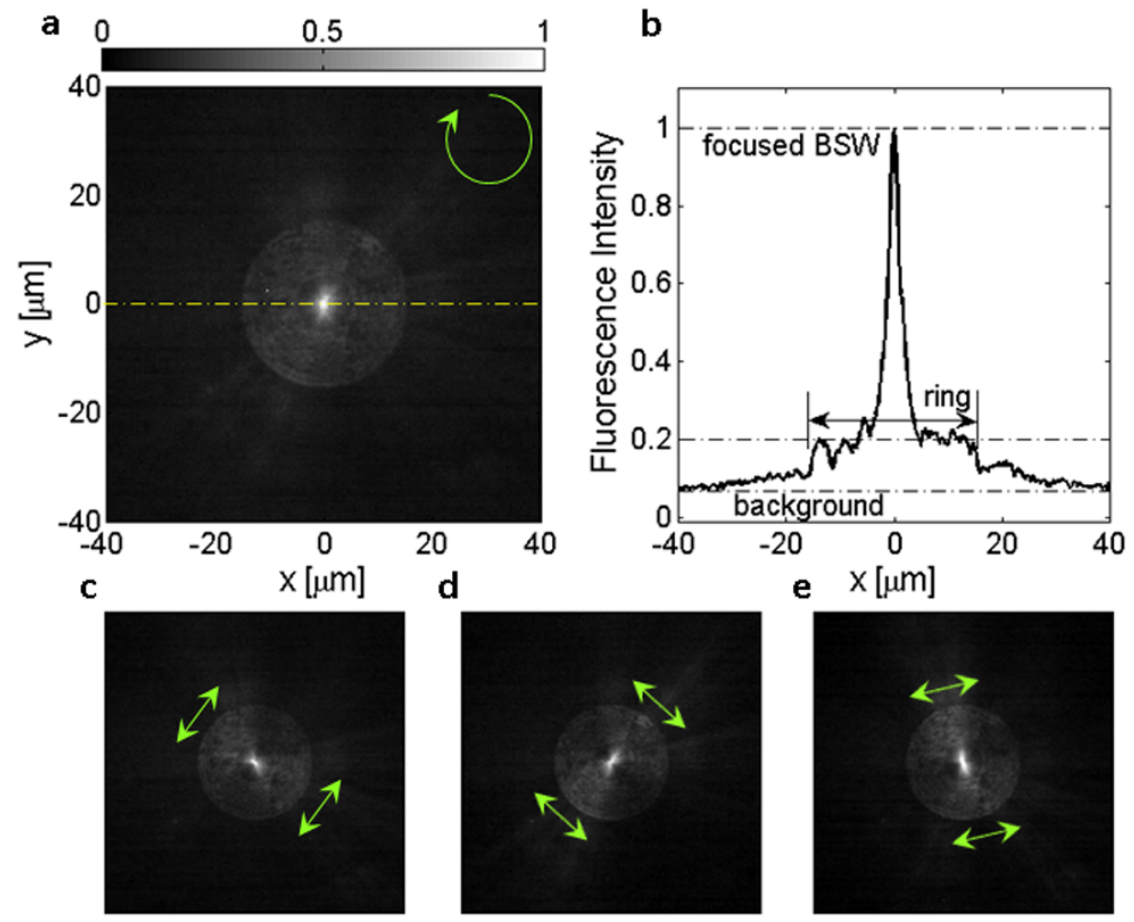

Figure 3 Fluorescence from focused BSW. (a), fluorescence image of a laser BSW coupled and focused by a circular grating $(\mathrm{D}=8 \mu \mathrm{m}, \Lambda=520 \mathrm{~nm})$ on "low leakage" multilayer. Illumination is a collimated, circularly polarized laser beam. (b), cross-sectional fluorescence intensity along the dashed line in (a). (c), (d), (e), same as in (a) with an incident laser beam linearly polarized as sketched by the arrows in the figures. All images are collected by means of the setup sketched in Supplementary Figure S5(b).

escence coupled into BSW is associated to the bright yellow ring with radius $\left[\left(\mathrm{k}_{\mathrm{x}} / \mathrm{k}_{0}\right)^{2}+\left(\mathrm{k}_{\mathrm{y}} / \mathrm{k}_{0}\right)^{2}\right]^{1 / 2} \approx 1.04$. The radius of the detected BSW-coupled fluorescence ring depends on the BSW dispersion curve $^{28}$. The other rings correspond to fluorescence coupled in other multilayer modes. Superposed to the BFP image, cross sectional intensity profiles (R-channel and G-channel) along an horizontal line at $\mathrm{k}_{\mathrm{y}} / \mathrm{k}_{0}=0$ are plotted. In addition, individual images corres- ponding to the R-channel and G-channel intensities are separately shown in a row on top of the RGB image. A polarization analysis shows that the BSW-coupled fluorescence has an inhomogeneous polarization that is azimuthally distributed ${ }^{28}$, as expected from the TE-polarization of BSW (see Supplementary Figure S6). A more detailed BFP-based analysis of BSW-coupled fluorescence from planar multilayers can be found at Ref. 20.

\section{a}
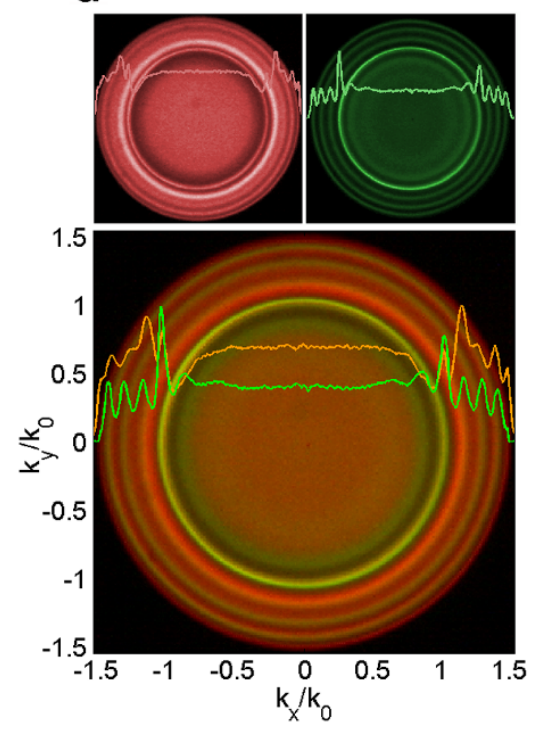

b
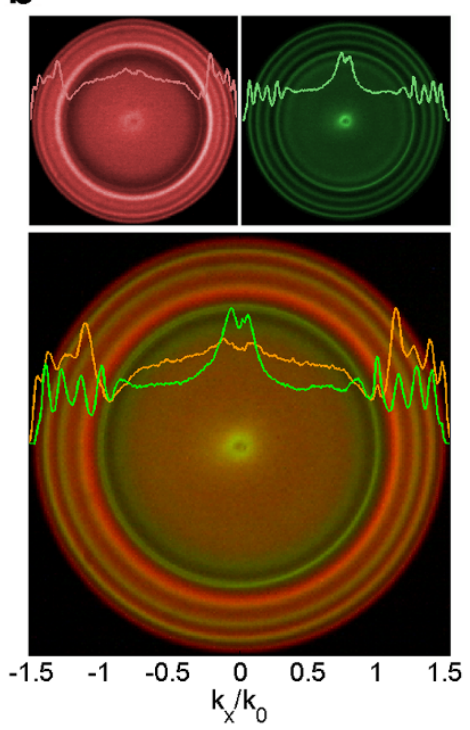

c

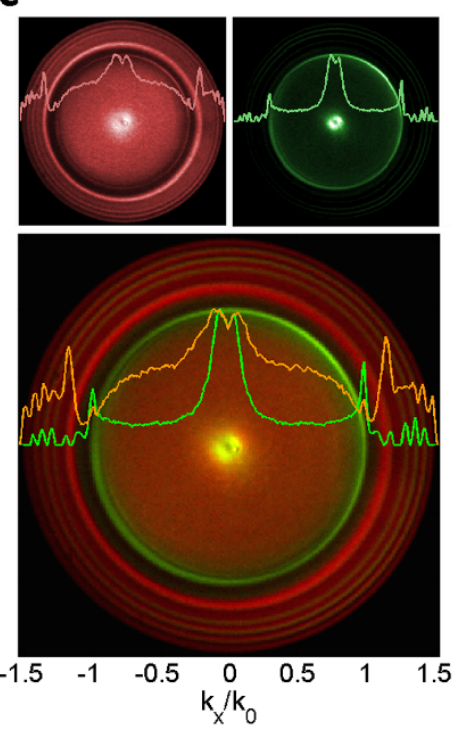

Figure 4 | Back Focal Plane images for BSW-coupled fluorescence. (a), fluorescence BFP images collected from a planar "regular" multilayer. (b), fluorescence BFP collected from a circular grating $(D=5 \mu \mathrm{m}, \Lambda=520 \mathrm{~nm})$ fabricated on "regular" multilayer. (c), fluorescence BFP collected from a circular grating $(\mathrm{D}=5 \mu \mathrm{m}, \Lambda=520 \mathrm{~nm})$ fabricated on "low leakage" multilayer. Illumination is a laser beam focused onto the center of the inner spacer. All images are collected by means of the setup sketched in Supplementary Figure S5(a). For each colour image, the corresponding R-channel (red) and Gchannel (green) images are separately shown in a row on top of the figure. 
When fluorescence is locally excited in the flat center of a grating spacer $(\mathrm{D}=5 \mu \mathrm{m}, \Lambda=520 \mathrm{~nm})$, a bright spot appears at $\mathrm{k}_{\mathrm{x}} / \mathrm{k}_{0}=0$, $\mathrm{k}_{\mathrm{y}} / \mathrm{k}_{0}=0$ in the corresponding BFP image, while the BSW-coupled fluorescence ring gets weaker (Fig. 4(b)). By illuminating the flat central area surrounded by the circular grating, we prevent the direct BSW coupling from the laser. The bright spot at $\mathrm{k}_{\mathrm{x}} / \mathrm{k}_{0}=0, \mathrm{k}_{\mathrm{y}} / \mathrm{k}_{0}=0$ is associated to fluorescence leaving the sample almost normally to the surface, with an estimated full divergence of less than 15 degrees. Thanks to a mechanism analogous to some plasmonic systems ${ }^{29,30}$, a portion of the energy emitted by the excited sources (characterized by randomly distributed dipolar momenta) is transferred to BSWs which in turn radially propagate away from the illuminated area. BSWs are then diffracted out of the multilayer by means of the circular grating, producing the observed beaming effect. In this respect, BSWs are mediating the transfer of energy from the localized sources to the free-space.

Unfortunately, as BSWs propagate onto the multilayer surface, some losses due to radiation leakage into the substrate occur. In order to prevent or minimize this unwanted effect, a "low leakage" multilayer has been employed (see Method section). Similarly to Fig. 4(b), Fig. 4(c) shows a fluorescence BFP image for a circular grating fabricated on a "low leakage" multilayer. Illumination is still provided by focusing the laser in the grating spacer center. After looking at the intensity contrast between the beamed central spot and the fluorescence coupled into other multilayers modes (including the BSW) or the fluorescence background, a general improvement of the beaming effect can be appreciated. Both the R-channel and the G-channel data indicate a significant increase in the light emitted along the multilayer normal direction, thanks to a reduction of losses in the energy-transfer mechanism mediated by BSWs.

The beaming effect can be largely improved if the circular grating is fabricated as an additive pattern on top of the last $\mathrm{SiO}_{2}$ layer of the stack. By properly playing with the dielectric loading mechanism (producing a redshift of the BSW dispersion curve ${ }^{19,31}$ ) and the circular grating period, we managed to fabricate an additive polymeric grating that significantly increases the amount of beamed fluorescence in a given spectral range. An exemplary result is illustrated in Supplementary Figure S7.

As discussed, the beaming effect arises from diffraction of BSWcoupled fluorescence propagating radially from the grating center, where multiple sources are excited. No such an effect can be observed if the circular symmetry is broken (i.e. the excitation laser spot is offcenter). In order to interpret the observed beaming in the BFP images, the following geometrical model is proposed. The circular grating can be considered as composed by multiple wedges of locally linear gratings, radially oriented. In a polar reference system centered in the spacer center, each wedge corresponds to a specific azimuthal angle. Assuming most of the radiated power as coupled to BSW, we can apply the Bragg law to each wedge, taking the fluorescent BSW as the incident radiation. On the Fourier plane, fluorescence diffracted on the first order $(+1)$ is distributed as a circular branch obtained by shifting the BSW-coupled fluorescence ring by an amount equal to the local grating vector module ${ }^{16}$. The direction of such a displacement is radial, and oriented according to the azimuthal angle of the specific wedge considered. As a result, multiple first-order $(+1)$ diffraction branches appear within the portion of the Fourier plane limited by the maximum NA of collection (NA $=1.49$ in the present case). The superposition of all diffraction branches leads to the appearance of a circular caustic in the Fourier plane, i.e. a geometrical locus wherein the diffracted power from each wedge accumulates. The size of the circular caustic depends on the grating vector $K=2 \pi /$ $\Lambda$. When $\mathrm{K}$ equals the fluorescent BSW wavevector (for a given wavelength), the caustic collapses into a single accumulation point, thus leading to a normally diffracted beaming with almost zero divergence. This model is very well supported by experimental observations. Several gratings with different periods $\Lambda$ have been fabri- cated and employed for BSW-assisted beaming. Results are shown in Fig. 5, together with the corresponding caustics calculated analytically on the Fourier plane.

When looking at the direct image of the 1DPC surface, we observe that the fluorescence beaming effect involves a diffraction mechanism occurring on the whole surface of the grating. BSW-coupled fluorescence can propagate radially from the sources excited in the inner spacer by the focused laser. Thanks to the low losses, BSW can propagates for long distances, and undergo diffraction by the grating Fig. 6(a)). Similarly to the direct plane images in Fig. 3, the collection optics used here has a low NA to prevent the contribution of substrate-leaking BSW-coupled fluorescence to the image (See Methods section). The measured intensity profile along a horizontal cross section shows a central maximum surrounded by a pair of symmetric, decreasing lobes. While the lobes are due to a BSW-assisted diffraction effect only, the intensity from the central peak is also due to fluorescence that is directly emitted in the free-space without any coupling to 1DPC modes (Fig. 6(b)). In particular, the lobe intensity drops quickly at the outer boundary of the circular grating, where no any further diffraction occurs. During the propagation across the grating, the BSW-coupled fluorescence undergoes losses mainly due to leakage and diffraction, and the observed fluorescence intensity becomes weaker and weaker as the grating outer boundaries are approached.

The plurality of sources excited by the focused laser have randomly oriented dipolar momentum, therefore the coupling to BSW can occur over all radial directions. However, the BSW are TE-polarized and this polarization state can be directly observed in the beamed fluorescence as well. Specifically, the diffracted fluorescence possesses an inhomogeneous polarization distribution depending on the azimuthal position of the local grating region it comes from. By inserting a polarization analyser along the collection path, the image in Fig. 6(a) is polarization filtered according to the analyser angular position, as shown in Fig. 6(c-e). This result confirms the $\mathrm{BSW}$-assisted characteristics of the beamed fluorescence. It should be recalled now that most of the fluorescence intensity observed in Fig. 6 is directed almost normally to the 1DPC surface, leading to a BSWassisted radiation extraction mechanism somehow complementary to the BSW focusing shown in Fig. 2 and Fig. 3.

Improvement in fluorescence collection at low Numerical Apertures. Results shown above suggest that the collection of fluorescence at low NA would be significantly improved thanks to the BSWassisted beaming effect. In this section we attempt a quantitative estimation of the fluorescence collection improvement.

In order to quantify the enhancement in fluorescence collection, we setup an original scanning microscope as illustrated in Fig. 7 and detailed in the Method section. Briefly, a scanning system is employed to locally laser-illuminate the sample surface and to feed the collected fluorescence into a spectrometer through a collection fiber. The fiber can be positioned on a BFP image plane, in such a way that only light having specific propagation directions is collected during the scan. Therefore, this system can provide image data with spatial, angular and spectral resolution for the collected fluorescence over a given raster scanned area. In the next, we present experimental results wherein the collection fiber collects fluorescence from a circular region centered at $\mathrm{k}_{\mathrm{x}} / \mathrm{k}_{0}=\mathrm{k}_{\mathrm{y}} / \mathrm{k}_{0}=0$ on the BFP and corresponding to a $\mathrm{NA} \approx 0.1$.

The false-color image in Fig. 8(a) is related to the collected fluorescence from a scan area containing the circular grating. Intensity values are integrated over a wavelength range from $\lambda=570 \mathrm{~nm}$ to $\lambda=595 \mathrm{~nm}$, after background subtraction. A significant increase of fluorescence is observed corresponding to the grating inner spacer, while the grating itself shows a faint fluorescence as compared to the background. This means that the BSW-assisted beaming effect improves the collection of fluorescence at $\mathrm{NA} \approx 0.1$ only when the 

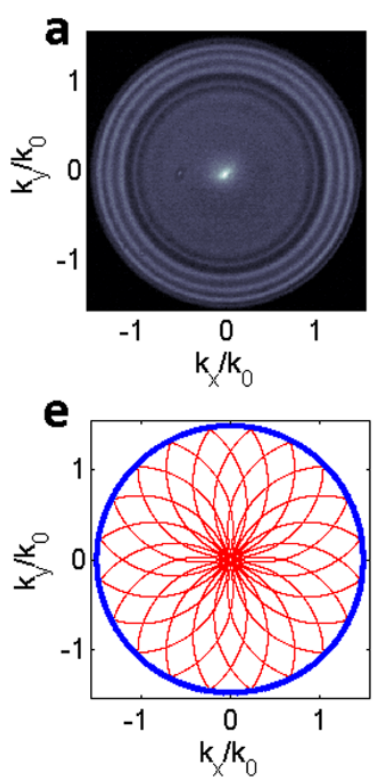
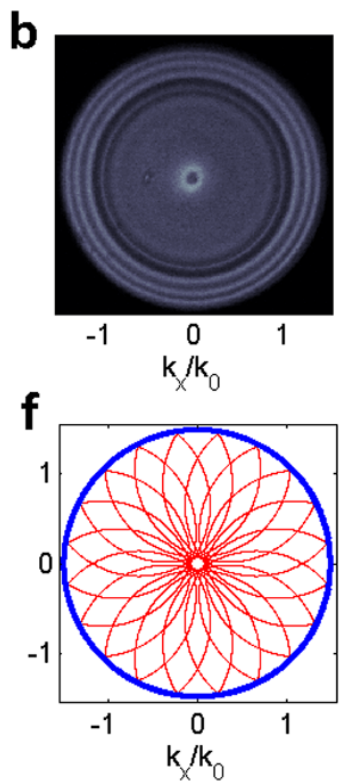
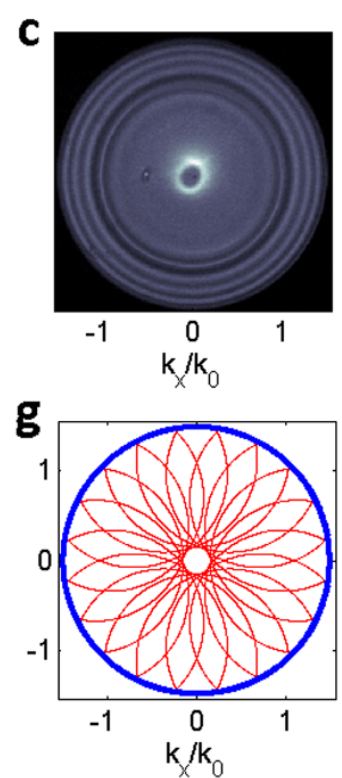

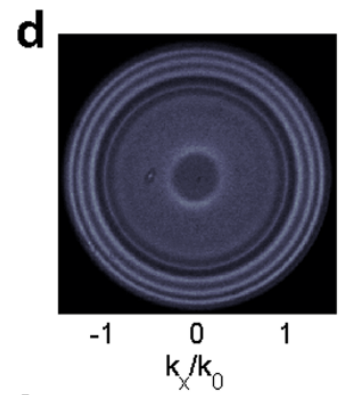

h

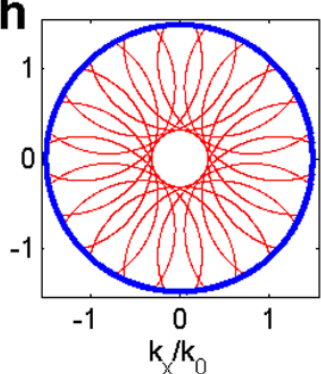

Figure 5 Caustics in BFP images. Different BFP images of circular gratings on "regular" multilayers are compared to corresponding schematic BFP patterns calculated by a simple geometrical model. All measured BFPs are obtained by locally exciting multiple fluorescent emitters in the center of several circular gratings $(\mathrm{D}=5 \mu \mathrm{m})$ with different periods $\Lambda$. The corresponding calculated patterns are obtained by applying the Bragg law to the BSW-coupled fluorescence ring as diffracted by multiple wedges of the circular grating. For each wedge, a circular branch associated to the first order diffracted BSW is drawn. The superposition of all those diffraction branches produces a caustic circle whereon all the diffracted intensities sum up. The diameter of the caustic circle depends on the grating period $\Lambda$ and indicates the angular shape of the diffracted fluorescence out of the multilayer surface. When the caustic circle collapses into a single point, the BSW-coupled fluorescence is almost completely beamed normally to the sample surface, with low divergence. (a), (e), grating period $\Lambda=560 \mathrm{~nm}$. (b), (f), grating period $\Lambda=520 \mathrm{~nm}$. (c), (g), grating period $\Lambda=500 \mathrm{~nm}$. (d), (h), grating period $\Lambda=440 \mathrm{~nm}$.

focused laser illuminates the spacer center, contrary to the case wherein the laser is focused elsewhere.

In Fig. 8(b), the emission spectrum from the inner spacer (integration over the black square in Fig. 8(a)) is compared to the emission spectrum from a region outside the structure (integration over the green square on top right corner in Fig. 8(a)). In order to quantify a collection enhancement factor due to the beaming effect, several spectral ranges are considered, as sketched in the Fig. 8(b). For each

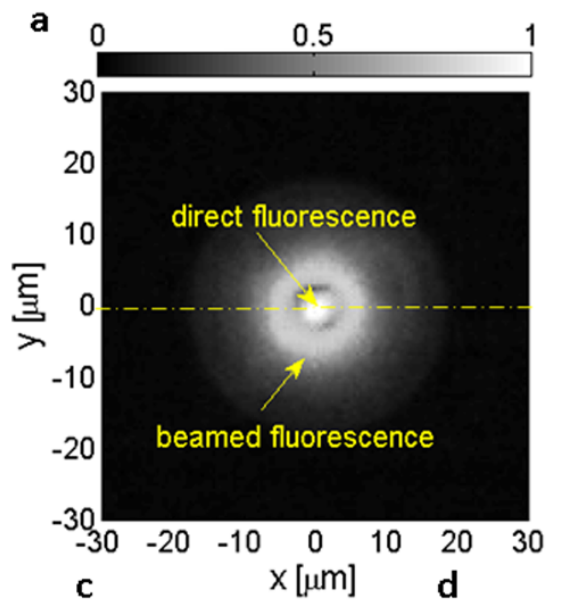

b
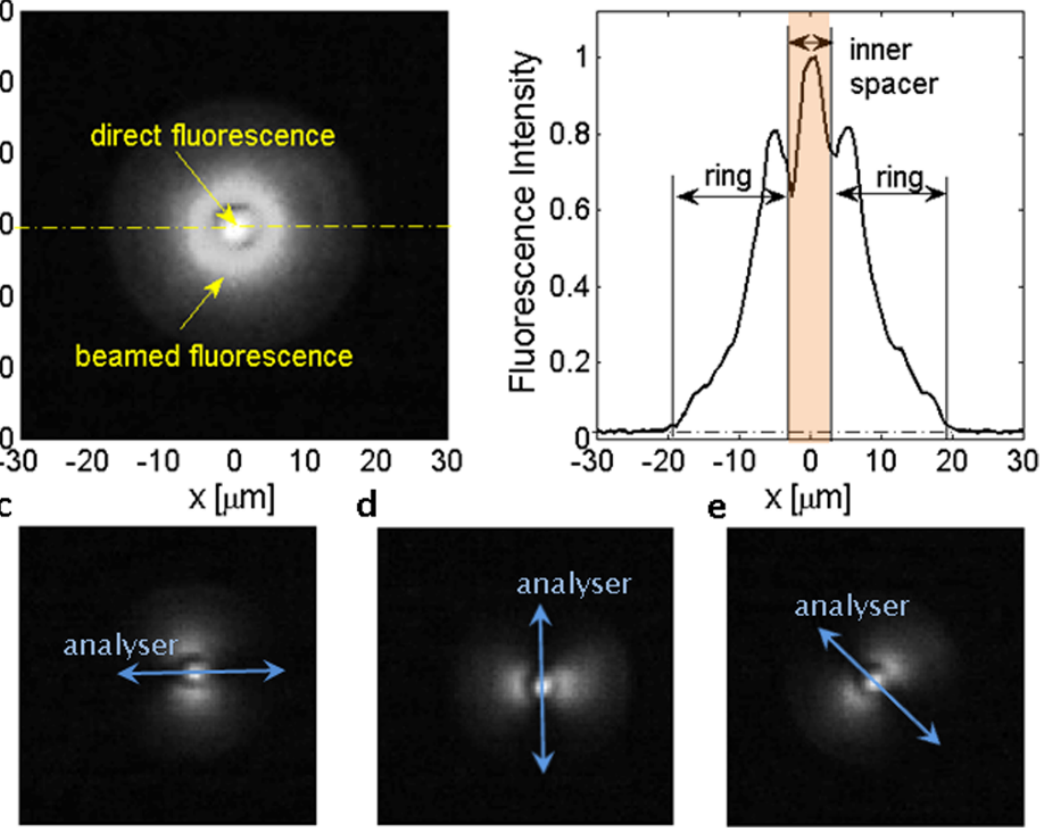

Figure 6 Spatial distribution of beamed BSW-coupled fluorescence. (a), direct image of fluorescence excited by a laser beam focused onto the flat inner spacer of a circular grating ( $\mathrm{D}=5 \mu \mathrm{m}, \Lambda=520 \mathrm{~nm}$ ) fabricated on "low leakage" multilayer. (b), cross-sectional fluorescence intensity along the horizontal dashed line in (a). (c), (d), (e), same as in (a) with the addition of a polarization analyser in collection, whose orientation is sketched by the arrows in the figures. All images are collected by means of the setup in Supplementary Figure S5(c). 


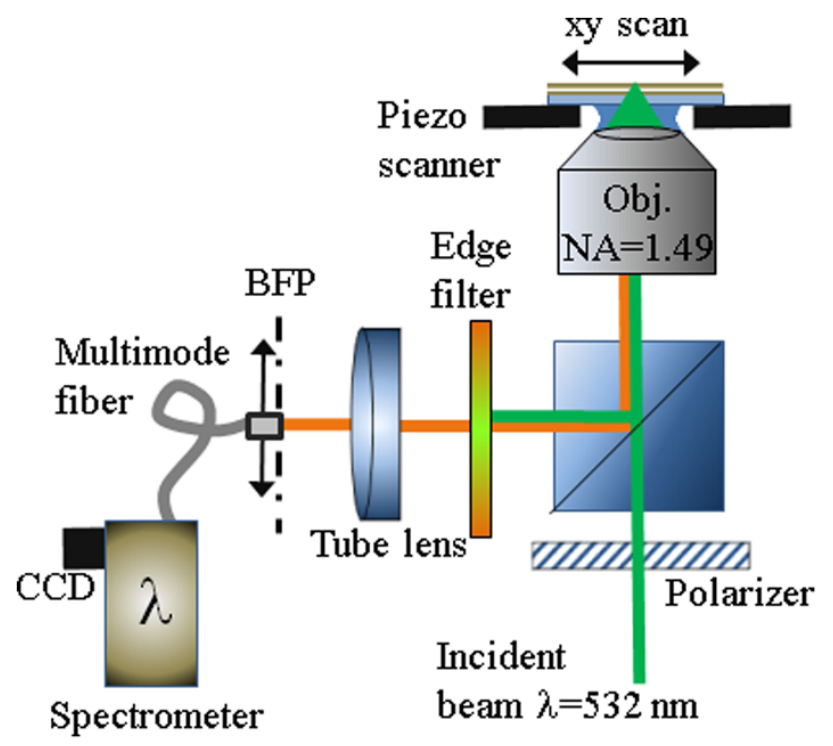

Figure 7 | BFP Scanning Fluorescence Microscope. The sample is scanned through a focused laser beam that locally excites fluorescence. Illumination is done by means of an oil immersion objective $(\mathrm{NA}=1.49)$. The radiation leaking into the substrate is collected by the same objective and spectrally filtered for having only fluorescence reaching the detector. A tube lens produces a magnified BFP image over a remote plane where a collection fiber (core diameter $50 \mu \mathrm{m}$ ) can be accurately positioned. The fiber is moveable along two directions, in such a way that light can be fibercollected from selected regions of the BFP image. Collected light is sent to a spectrometer equipped with a CCD camera. As an example, when the fiber is placed in the very center of the BFP image, only light propagating normally with respect to the sample surface $\left(\mathrm{k}_{\mathrm{x}}=0, \mathrm{k}_{\mathrm{y}}=0\right)$ is collected and spectrally analysed. Since the illumination is focused, it is therefore possible to obtain spatially resolved spectral information about the fluorescence leaving the sample along specific directions of propagation.

spectral range, the ratio $\mathrm{I}_{\text {spacer }} / \mathrm{I}_{\text {outside }}$ is calculated, wherein $\mathrm{I}_{\text {spacer }}$ and $\mathrm{I}_{\text {outside }}$ refer to the fluorescence intensities integrated over the corresponding spectral range and over spatial regions defined by the black box and the green box in Fig. 8(a), respectively. A set of values for the ratio $I_{\text {spacer }} / I_{\text {outside }}$ is obtained by making the green box sequentially sampling the whole image, with the exclusion of the grating area. Finally, the collected data set for each spectral range are represented as histograms, as shown in Fig. 8(c-g). Each histogram provides information about the statistical distribution of the enhancement factor, clearly wavelength-dependent. For example, the highest enhancement factor is estimated as $26.37 \pm$ 10.93 and it is observed for wavelengths between $582.5 \mathrm{~nm}$ and $588.75 \mathrm{~nm}$ (Fig. 8(e)). If the total wavelength range from $\lambda=$ $570 \mathrm{~nm}$ to $\lambda=595 \mathrm{~nm}$ is considered, the enhancement factor is estimated as $19.18 \pm 5.36$ (Fig. $8(\mathrm{~g})$ ).

\section{Discussion}

In this work we demonstrate that BSW on 1DPC can play the role of mediator of energy transfer from localized volumes to free space and vice versa. For example, a grating coupler can focus BSW at the 1DPC/air interface on a region smaller than the diffraction limit for propagating light in air. The surface-bound nature of BSW is such that the field intensity reaches its maximum substantially at the 1DPC surface. Although such an all-dielectric approach is not suitable to attain to a deep subwavelength localization of electromagnetic energy, as in many nano-plasmonic arrangements, BSW focusing represents a real three dimensional confinement of radiation over a region that is substantially planar and several microns apart from the coupling structure.
By reciprocity, emitters located on the 1DPC surface are likely to couple a significant portion of energy to BSW, despite the inherently multimodal features of the $1 \mathrm{DPC}^{32}$. This preferential coupling can also be ascribed to the surface-bound nature of BSW. Once radiated energy is transferred to BSW, it can be manipulated with diffraction gratings, as shown in the results presented above. Other arrangements can be used as well, including waveguides ${ }^{19}$, refractive elements $^{33}$, and so on. Unlike the majority of photonic crystal structures, the surface modes are here sustained without the need of any surface corrugation ${ }^{34}$. Therefore, BSW can be further manipulated by fabricating suitable elements on a basically flat surface, thus easing the overall fabrication process.

The use of BSW as energy carrier presents some advantages as compared, for example, to the surface plasmon counterpart. First, losses due to absorption are dramatically reduced thanks to the use of dielectric materials. This effect produces direct benefits in terms of surface wave propagation length, resonance quality factor and nearfield enhancement on the 1DPC surface as well. Furthermore, BSW spectral range (from UV to near infrared), dispersion and polarization state (either TE or TM) can be almost arbitrarily tuned by operating on the 1DPC design ${ }^{35-37}$.

In conclusion, we provide here an all-dielectric platform showing an inherent surface-resonant behavior ${ }^{38}$ that can be exploited for energy transfer over large distances. Although we presented a specific illustrative example of some BSW-mediated effects, the underlying principle can be widely applied to other arrangements. For example, plasmonic nano-structures such as nano-antennas or nano-particles can be integrated on a 1DPC surface, in a so-called hybrid configuration $^{39}$ in such a way that further field confinement effects can be produced. The use of dielectric multilayers is compatible with most of the fabrication technology currently employed for optical nanoantennas on transparent substrates. Therefore, it would be possible to surround the plasmonic nano-objects with a photonic-tailored environment that can ease the coupling of energy (e.g. elastic scattering, fluorescence, Raman) to/from the outer world, mediated by $\mathrm{BSW}^{40-43}$. The improvements from this platform can be readily appreciated in a number of surface-optics application fields such as lens-free optical sensing ${ }^{44,45}$ and lighting ${ }^{46,47}$.

\section{Methods}

Sample fabrication. The 1DPC consists of a dielectric multilayer made of a stack of $\mathrm{Ta}_{2} \mathrm{O}_{5}$ (high refractive index) and $\mathrm{SiO}_{2}$ (low refractive index) layers, deposited on a glass coverslip $150 \mu \mathrm{m}$ thickness by plasma ion assisted deposition under high vacuum conditions (APS904 coating system, Leybold Optics). A schematic of the sample is presented in the Fig. 1 . The stack sequence is glass- $\left[\mathrm{Ta}_{2} \mathrm{O}_{5}-\mathrm{SiO}_{2}\right] \times 6$ $\mathrm{Ta}_{2} \mathrm{O}_{5}-\mathrm{SiO}_{2}$-air for the "regular" layout, while we have glass- $\left[\mathrm{Ta}_{2} \mathrm{O}_{5}-\mathrm{SiO}_{2}\right] \times 10$ $\mathrm{Ta}_{2} \mathrm{O}_{5}-\mathrm{SiO}_{2}$-air for the "low leakage" layout. Therefore, the "regular" multilayer has a total of 16 layers, while the "low-leakage" multilayer keeps the same stack sequence but has 22 layers in total. The $\mathrm{Ta}_{2} \mathrm{O}_{5}$ layer is $95 \mathrm{~nm}$ thick, the $\mathrm{SiO}_{2}$ layer is $137 \mathrm{~nm}$ thick, but the latest $\mathrm{SiO}_{2}$ layer on top of the stack is only $127 \mathrm{~nm}$ thick. The ring structure is fabricated by electron beam lithography followed by a Reactive Ion Etching step. The geometrical parameters are detailed in the Fig. 1. For fluorescencebased measurements, a homogeneous thin layer of protein A labelled with AlexaFluor 546 at a concentration of $3 \mu \mathrm{g} / \mathrm{ml}$ is incubated on the sample surface and then washed. Due to electrostatic interaction, the fluorescent protein is homogeneously grafted on the sample surface, thus providing a very thin layer of fluorescent material. Having a uniform distribution of emitters on the sample surface raises some advantages. Indeed, it allows to locally excite fluorescence in any location, by simply focusing an excitation laser beam where needed. In addition, when the illumination is distributed over a large area, intensity contrast comparisons are readily accessible by a simple offline analysis of wide-field images.

The planar multilayer sustains BSW having a dispersion curve as illustrated by the calculated reflectance map shown in Supplementary Figure S1(a). The calculated BSW intensity profile at a wavelength $\lambda=532 \mathrm{~nm}$ shows a 600 fold enhancement on the multilayer surface, as illustrated in Supplementary Figure S1(b).

Leakage radiation interference microscopy setup. The experimental setup is sketched in Supplementary Figure S2. A detailed description of the working principle of the interferometric technique employed for the amplitude and phase retrieval in this arrangement can be found elsewhere ${ }^{48}$. A collimated beam from a frequency doubled Nd:YAG laser source at $\lambda=532 \mathrm{~nm}$ is split in two arms of a Mach-Zehnder interferometer by means of a Polarizing Beam Splitter (PBS). The polarization of the 


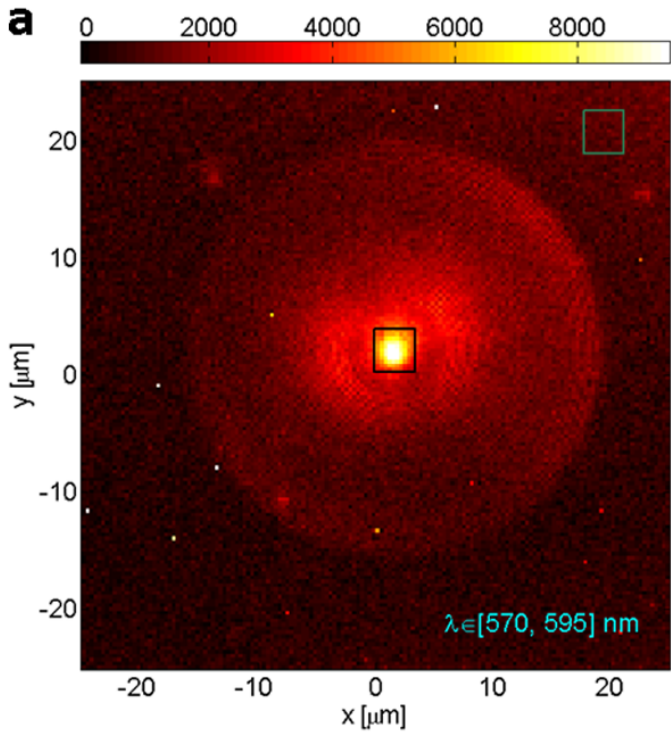

b
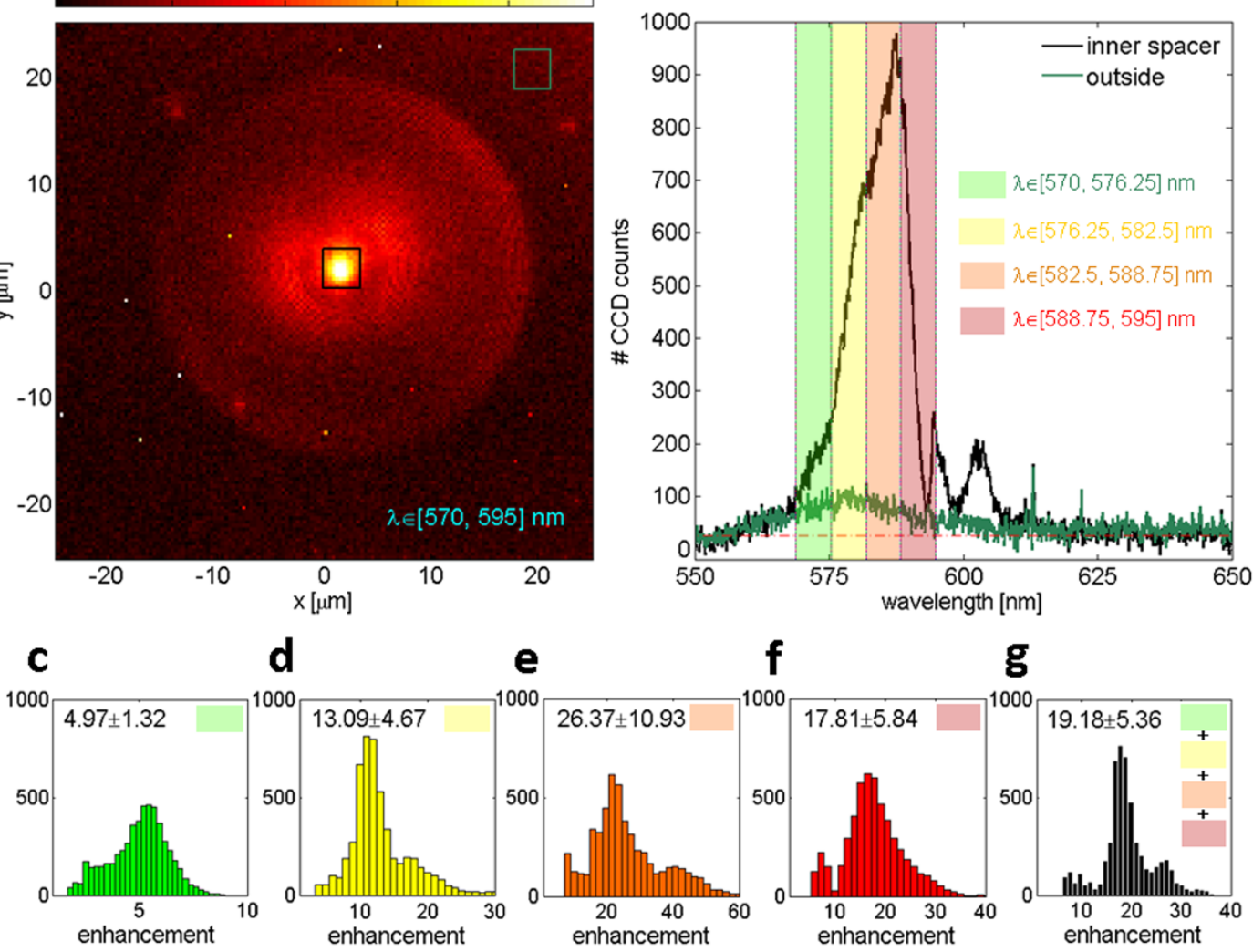

Figure $8 \mid$ Spectroscopic imaging of beamed BSW-coupled fluorescence. (a) False-color image of the circular grating as obtained by means of the BFP scanning system. Intensity values are integrated over a spectral range from $\lambda=570 \mathrm{~nm}$ to $\lambda=595 \mathrm{~nm}$. (b) Fluorescence spectra corresponding to the grating inner spacer and outside the grating. Coloured bars indicate spectral intervals used for further analysis. (c-g) statistical distributions of the enhancement factor calculated over the corresponding spectral ranges. Average and standard deviation are indicated for each spectral range.

light beams can be independently controlled through a pair of half wavelength plates and Glan-Taylor polarizers. The object beam is used for illumination and sent to an objective (Numerical Aperture, $\mathrm{NA}=0.5$ ) used for sample illumination. The effective NA of the illumination objective can be reduced by operating on a diaphragm in front of the objective entrance pupil. In this way, the angular spread of the incident laser beam can be tuned in order to satisfy the momentum matching required for BSW grating-coupling.

Leakage radiation emerging from the sample is collected with an oil immersion objective (Nikon APO TIRF 100 $\times, \mathrm{NA}=1.49$ ) and imaged through a tube lens on a Charge-Coupled Device (CCD), where it is superposed with the reference beam. The phase of the reference beam is changed from 0 to $2 \pi$ in five steps by means of a piezoactuated mirror. The five corresponding images are fed into an offline image processing algorithm based on the Schwider-Hariharan method $^{49}$ and amplitude and phase distributions of the collected field (leakage radiation) are then retrieved. In order to collect the leakage radiation only, a beam blocker has been inserted after the collection objective in such a way that the light propagating inside the light cone in air $(\mathrm{NA} \leq 1)$ is filtered out.

Fluorescence imaging systems. Fluorescence images are collected by employing two apparatuses. For the Back Focal Plane (BFP) measurement, a colour RGB CMOS camera (DCC1645C from Thorlabs) is used. The setup is sketched in Supplementary Figure S5(a) and has an upper illumination stage and a lower collection stage. In the measurements presented here, the upper stage mounts a $\mathrm{NA}=0.2$ objective (Zeiss Ultrafluar $10 \times$ ) working in air. The lower stage mounts a NA $=1.49$ oil immersion objective with (Nikon APO TIRF 100 $\times$ ). The illumination objective focuses a doubled frequency Nd:YAG laser beam $(\lambda=532 \mathrm{~nm})$ onto the sample surface. The laser beam is obtained by collimating the output of a single-mode fiber wherein a freespace propagating beam has been injected from the laser cavity. In order to filter out the Raman signal coming from the fiber, a laser line filter is employed after the collimation of the beam (532 nm MaxLine ${ }^{\circledR}$, Semrock). Upon illumination, the light leaking into the substrate is collected by the oil immersion objective and then spectrally filtered (RazorEdge ${ }^{\circledR}$ Longpass 532 , Semrock) in order to block the laser radiation. A tube lens produces a BFP image of the collection objective onto the camera. Thanks to this feature, the angular distribution of fluorescence leaking into the substrate can be imaged. For the direct imaging of the multilayer surface (e.g. as shown in Fig. 3 and Fig. 5), a monochromatic CCD camera (Apogee Ascent) is employed, as shown in Supplementary Figures S5(b,c). Most of the setup is analogous to the previous one, but the laser illumination from the upper objective is distributed as a uniform beam over a circular region $150 \mu \mathrm{m}$ wide. This homogeneous illumination can be obtained by feeding the illumination objective with a laser beam weakly focused on its back focal plane, in such a way that the objective operates as a collimator. In alternative, illumination can be a focused laser beam (Supplementary Figure S5(c)). Fluorescence is collected by a NA $=0.2$ objective operating in air, in such a way that only the directly emitted fluorescence and the beamed BSW-coupled fluorescence contribute to the final image recorded on the camera, therefore excluding the BSW-coupled fluorescence leaking into the substrate.

BFP Scanning fluorescence microscope. The setup is aimed at performing a spectral analysis of light emitted from the sample surface with both spatial and angular resolution. As sketched in Fig. 7, the sample is scanned through a focused laser beam $(\lambda=532 \mathrm{~nm})$ emerging from an oil immersion objective $(\mathrm{NA}=1.49)$. The leakage radiation is collected by means of the same objective and then passed through an edge filter (RazorEdge ${ }^{\circledR}$ Longpass 532) to filter out the laser radiation. A tube lens produces a magnified BFP image of the collection objective onto a remote plane, wherein a multimode fiber end is located. The fiber end is supported by a two-dimensional translational stage, and can be accurately moved onto the BFP image plane. The fiber collects fluorescence from specific portions on the BFP image and delivers the collected radiation to a spectrometer (Acton SpectraPro 300, Princeton Instruments). Since the angular resolution achievable with such a system is inversely proportional to the lateral dimension of the collection fiber core, we found a tradeoff between fiber diameter, BFP magnification factor and signal-to-noise ratio of fluorescence spectra as measured by the spectrometer CCD. In a suitable configuration, the fiber diameter is completely contained within a region of NA $\leq 0.1$ on the BFP image plane. During the scan, fluorescence is locally excited by the focused laser spot. For each scan position, the fluorescence spectrum can be recorded, according to the specific propagation direction as determined by positioning the collection fiber onto the BFP image. The presented measurement consists of an array of $150 \times 150$ pixels, with an integration time of $5 \mathrm{~ms}$ for each pixel. The laser illumination is kept at low power to avoid rapid photobleaching of the emission during the scan.

1. Novotny, L. \& Van Hulst, N. F. Antennas for light. Nat. Photon. 5, 83-90 (2011).

2. Liu, Z. et al. Focusing surface plasmons with a plasmonic lens. Nano Lett. 5, 1726 (2005). 
3. Kosako, T., Kadoya, Y. \& Hofmann, H. F. Directional control of light by a nanooptical Yagi-Uda antenna. Nat. Photon. 4, 312-315 (2010).

4. Coenen, T., Vesseur, E. J., Polman, A. \& Koenderink, F. A. Directional emission from plasmonic Yagi-Uda antennas probed by angle-resolved cathodoluminescence spectroscopy. Nano Lett. 11, 3779-84 (2011).

5. Curto, A. G. et al. Unidirectional emission of a quantum dot coupled to a nanoantenna. Science 329, 930-933 (2010).

6. Taminiau, T. H., Stefani, F. D., Segerink, F. B. \& Van Hulst, N. F. Optical antennas direct single-molecule emission. Nat. Photon. 2, 234-237 (2008).

7. Schoen, D. T., Coenen, T., Garcia de Abajo, F. J., Brongersma, M. L. \& Polman, A. The planar parabolic optical antenna. Nano Lett. 13, 188-193 (2013).

8. Dregely, D. et al. 3D optical Yagi-Uda nanoantenna array. Nat. Comm. 2, 267 (2011).

9. Langguth, L., Punj, D., Wenger, J. \& Koenderink, F. A. Plasmonic band structure controls single-molecule fluorescence. ACS Nano 7, 8840-8848 (2013).

10. Li, H. et al. Tunable plasmons in shallow silver nanowell arrays for directional surface-enhanced raman scattering. J. Phys. Chem. C. 116, 23608-15 (2012).

11. Li, H. et al. Active plasmonic nanoantennas for controlling fluorescence beams. J. Phys. Chem. C. 117, 19154-59 (2013).

12. Baron, A. et al. Compact antenna for efficient and unidirectional launching and decoupling of surface plasmons. Nano Lett. 11, 4207 (2011).

13. Zhao, C., Liu, Y., Zhao, Y., Fang, N. \& Huang, T. J. A reconfigurable plasmofuidic lens. Nat. Comm. 4, 2305 (2013).

14. Lerman, G. M. \& Levy, U. Pin cushion plasmonic device for polarization beam splitting, focusing and beam position estimation. Nano Lett. 13, 1100-1105 (2013)

15. Zentgraf, T., Liu, Y., Mikkelsen, M. H., Valentine, J. \& Zhang, X. Plasmonic luneburg and eaton lenses. Nat. Nano. 6, 151-155 (2011).

16. Offerhaus et al. Creating focused plasmons by noncollinear phasematching on functional gratings. Nano Lett. 5, 2144-2148 (2005).

17. Jun, C., Huang, K. C. Y. \& Brongersma, M. Plasmonic beaming and active control over fluorescent emission. Nat. Comm 2, 283 (2011).

18. Aouani, H. et al. Plasmonic antennas for directional sorting of fluorescence emission. Nano Lett. 11, 2400-2406 (2011).

19. Descrovi, E. et al. Guided Bloch surface waves on ultrathin polymeric ridges. Nano Lett. 10, 2087-2091 (2012).

20. Badugu, R., Nowaczyk, K., Descrovi, E. \& Lakowicz, J. R. Radiative Decay engineering 6: fluorescence on one-dimensional photonic crystal. Anal. Biochem. 442, (2013).

21. Descrovi, E. et al. Leakage radiation interference microscopy. Opt. Lett. $\mathbf{3 8}$ 3374-3376 (2013)

22. Lerman, G. M., Yanai, A. \& Levy, U. Demonstration of nanofocusing by the use of plasmonic lens illuminated with radially polarized light. Nano Lett. 9, 2139-2143 (2009).

23. Zhaowei, L. et al. Focusing surface plasmons with a plasmonic lens. Nano Lett. 5 , 1726-1729 (2005).

24. Kim, J. et al. Experimental and theoretical study of the Gouy phase anomaly of light in the focus of microlenses. J. Opt. 15, 105708 (2013).

25. Ballarini, M. et al. Bloch surface waves-controlled emission of organic dyes grafted on a one-dimensional photonic crystal. Appl. Phys. Lett. 99, 043302 (2011)

26. Zhang, D. G., Yuan, X. C., Yuan, G. H., Wang, P. \& Ming, H. Directional fluorescence emission characterized with leakage radiation microscopy. J. Opt. 12 035002 (2010).

27. Sersic, I., Tuambilangana, C. \& Koenderink, A. F. Fourier microscopy of single plasmonic scatterer. New J. Phys. 13, 083019 (2011)

28. Angelini, A. et al. Fluorescence diffraction assisted by Bloch surface waves on a one-dimensional photonic crystal. New J. Phys. 15, 073002 (2013).

29. Rui, G., Abeysinghe, D. C., Nelson, R. L. \& Zhan, Q. Demonstration of beam steering via dipole-coupled plasmonic spiral antenna. Sci. Rep. 3, 22372013 (2013)

30. Aouani, H. et al. Bright unidirectional emission of molecules in a nanoaperture with surface corrugations. Nano Lett. 11, 637-644 (2011).

31. Lu, H. et al. Polymer-loaded propagating modes on a one-dimensional photonic crystal. Appl. Phys. Lett. 104, 061115 (2014).

32. Zhang, D. et al. Back focal plane imaging of directional emission from dye molecules coupled to one-dimensional photonic crystals. Nanotechnology $\mathbf{2 5}$ 145202 (2014)

33. Yu, L. et al. Manipulation Bloch surface waves in 2D: a platform concept based flat lens. Light: Sci. Appl. 3, e124 (2014).

34. Ganesh, N. et al. Enhanced fluorescence emission from quantum dots on a photonic crystal surface. Nat. Nano. 2, 515-520 (2007).
35. Descrovi, E., Giorgis, F., Dominici, L. \& Michelotti, F. Experimental observation of optical bandgaps for surface electromagnetic waves in a periodically corrugated one-dimensional silicon nitride photonic crystal. Opt. Lett. 33, 243-245 (2008).

36. Farmer, A., Friedli, C. A., Wright, S. M. \& Robertson, W. M. Biosensing using surface electromagnetic waves in photonic bandgap multilayers. Sens. Actuators $B$ 173, 79-84 (2012).

37. Sreekanth, K. V., Zeng, S., Shang, J., Yong, K. T. \& Yu, T. Excitation of surface electromagnetic waves in a graphene-based Bragg grating. Sci. Rep. 2, 737 (2012).

38. Lee, K. G. et al. A planar dielectric antenna for directional single-photon emission and near-unity collection efficiency. Nat. Photon. 5, 166-169 (2011).

39. Chanda, D. et al. Coupling of plasmonic and optical cavity modes in quasi-threedimensional plasmonic crystals. Nat. Comm. 2, 479 (2011).

40. Wang, D., Yang, T. \& Crozier, K. B. Optical antennas integrated with concentric ring gratings: electric field enhancement and directional radiation. Opt. Express 19, 2148 (2011)

41. Ahmed, A. \& Gordon, R. Directivity enhanced Raman spectroscopy using nanoantennas. Nano Lett. 11, 1800-1803 (2011).

42. Pirotta, S. et al. Surface-Enhanced Raman Scattering in Purely Dielectric Structures via Bloch Surface Waves. J. Phys. Chem. C 117, 6821-6825 (2013).

43. Schmidt, M. A., Lei, D. Y., Wondraczek, L., Nazabal, V. \& Maier, S. Hybrid nanoparticle-microcavity-based plasmonic nanosensors with improved detection resolution and extended remote-sensing ability. Nat. Comm. 3, 1108 (2012).

44. Cetin, A. E. et al. Handheld high-throughput plasmonic biosensor using computational on-chip imaging. Light: Sci. Appl. 3, e122 (2014).

45. Toma, M. et al. Surface plasmon-coupled emission on plasmonic Bragg gratings. Opt. Express 20, 14042-14053 (2012).

46. Choy, J. T. et al. Spontaneous emission and collection efficiency enhancement of single emitters in diamond via plasmonic cavities and gratings. Appl. Phys. Lett. 103, 161101 (2013)

47. Lozano, G. et al. Plasmonics for solid-state lighting: enhanced excitation and directional emission of highly efficient light sources. Light: Sci. Appl. 2, e66 (2013).

48. Kim, M. S., Scharf, T., Etrich, C., Rockstuhl, C. \& Herzig, H. P. Longitudinaldifferential interferometry: direct imaging of axial superluminal phase propagation. Opt. Lett. 37, 305-307 (2012).

49. Hariharan, P. P., Oreb, B. F. \& Eiju, T. Digital phase-shifting interferometry: a simple error-compensating phase calculation algorithm. Appl. Opt. 26, 25042056 (1987).

\section{Acknowledgments}

This research has received funding from the Italian Flagship Project NANOMAX (Progetto Bandiera MIUR PNR 2011-2013), the Italian FIRB 2011 NEWTON (RBAP11BYNP), the Swiss National Science Foundation (SNSF) and the EU FP7 project BILOBA (Grant \# 318035).

\section{Author contributions}

E.D. conceived the idea and wrote the manuscript. A.A. performed calculations and fluorescence measurements. E.B. performed the interferometric measurements. P.M. performed the multilayer deposition. L.B., N.D.L. and E.E. developed the fabrication process for ring structures on multilayers. C.F.P., F.G. and H.P.H. supervised the activities. All authors contributed to the scientific discussion and revision of the article.

\section{Additional information}

Supplementary information accompanies this paper at http://www.nature.com/ scientificreports

Competing financial interests: The authors declare no competing financial interests. How to cite this article: Angelini, A. et al. Focusing and Extraction of Light mediated by Bloch Surface Waves. Sci. Rep. 4, 5428; DOI:10.1038/srep05428 (2014).

This work is licensed under a Creative Commons Attribution-NonCommercialNoDerivs 4.0 International License. The images or other third party material in this article are included in the article's Creative Commons license, unless indicated otherwise in the credit line; if the material is not included under the Creative Commons license, users will need to obtain permission from the license holder in order to reproduce the material. To view a copy of this license, visit http:// creativecommons.org/licenses/by-nc-nd/4.0/ 